\title{
REVIEW
}

\section{Management of osteoporosis in men: an update and case example}

\author{
Aliya A. Khan, Anthony B. Hodsman, Alexandra Papaioannou, David Kendler, Jacques P. Brown, \\ Wojciech P. Olszynski
}

\section{ABSTRACT}

In 2002, Osteoporosis Canada published clinical practice guidelines for the diagnosis and management of osteoporosis. The current paper supplements that guideline and provides a review and synthesis of the current literature on the diagnosis and management of osteoporosis in men.

CMAJ 2007:176(3):345-8

The Case: A 66-year-old, previously well, retired male firefighter arrives with back pain and long-standing asthma requiring intermittent oral glucocorticoid use over the past 15 years. He estimates that he is about $6 \mathrm{~cm}$ shorter than he was 10 years ago. He has no family history of osteoporosis. He is physically active, drinks 2 servings of alcoholic beverages per day and quit smoking 5 years earlier. His physical exam is unremarkable except for a mild dorsal kyphosis. How should this (hypothetical) case be investigated and managed?

$\mathrm{T}$ hat osteoporosis affects men as well as women is often underappreciated. The reasons that women are more frequently affected than men are several: men achieve a higher peak bone mass, experience lower rates of bone loss, lack a menopausal equivalent and have a shorter life expectancy. Differences in patterns of bone loss over time also contribute to a biomechanical advantage among men, with aging. Although men, like women, experience enhanced endocortical bone resorption with age, men's periosteal bone formation is greater in volume. This enhanced periosteal bone formation results in a greater cross-sectional bone diameter and a biomechanical advantage, as larger bones have less risk of fracture. ${ }^{1}$ Nevertheless, vertebral deformities, which often represent vertebral fractures, are seen as often in men as in women according to the Canadian Multicentre Osteoporosis Study (CaMos), a population-based sample of healthy men and women living in the community. CaMos found that the prevalence of vertebral deformities in subjects over the age of 50 years was $21.5 \%$ among men and $23.5 \%$ among women. ${ }^{2}$ It has been hypothesized that the presence of I or 2 vertebral fractures may have been the result of heavy physical labour or previous trauma. Multiple vertebral fractures, however, appear to be associated with risk factors for osteoporosis, and increase with age in a pattern similar to that seen in women. Multiple vertebral fractures in men are therefore likely to be caused by underlying osteoporosis. Although the majority of hip fractures $(73 \%)$ occur to women, when men break a hip they are more likely than women to die or experience disability. ${ }^{3}$

Osteoporosis Canada ${ }^{4}$ and the World Health Organization (WHO) have identified a series of factors that increase the risk for osteoporotic fractures (Box I), independent of the degree to which bone mineral density (BMD) is reduced. ${ }^{5}$

\section{The role of bone densitometry}

As in the 2002 guidelines, BMD testing for all men over 65 is advised, since low bone density contributes significantly to the future risk for skeletal fractures in this age group. BMD testing is also advised for younger men in the presence of secondary causes of osteoporosis and other risk factors for fracture (Box 2).

Box 1: Factors independent of bone mineral density that increase fracture risk in men

Primary factors

- Previous fragility fracture after 40 years of age, especially vertebral compression fractures*

- Systemic glucocorticoid therapy $\dagger$ of $\geq 3$ months' duration

- Advancing age, especially past $65 \mathrm{yr}$

Other key factors

- Presence of disease or a condition associated with bone loss

- Family history of osteoporosis; fracture in a parent

- High alcohol intake: $>2$ units (i.e., $>18 \mathrm{~g}$ ) of alcohol daily

- Hypogonadism, primary or secondary

- Low BMI $\left(<20 \mathrm{~kg} / \mathrm{m}^{2}\right)$ - associated with bone loss

- Smoking, current or past

- Use of LHRH analogs (anti-androgen therapy)

$\mathrm{LHRH}=$ luteinizing hormone-releasing hormone, $\mathrm{BMI}=$ body mass index. *Height loss of $\geq 6 \mathrm{~cm}$ or kyphosis may be a clinical sign of a vertebral compression fracture.

†The equivalent of $\geq 7.5 \mathrm{mg} / \mathrm{d}$ of prednisone. 
In men over 50 years of age, a diagnosis of osteoporosis may be considered when the T-score is -2.5 or less. The original WHO diagnostic classification, however, was intended to apply to postmenopausal women over 50 years of age, and should not be applied to younger people. In men younger than 50 years, it is recommended that simple $z$ scores be used to describe the degree to which the bone-density measurement differs from normal: $z$ scores less than -2 are below the expected range for age. Moreover, "osteopenia" should no longer be considered as a diagnosis. Instead, patients over 50 years of age with T-scores between -2.5 and $-\mathrm{I} .5$ should be described as having "reduced bone density" and a diagnosis of osteoporosis made only in the presence of a fragility fracture. Table I lists the diagnostic classifications currently recommended by Osteoporosis Canada. It is important to note that people with reduced bone density may not necessarily be at an increased risk of fracture; such patients should be further assessed, with stratification of their fracture risk.

\section{Identifying absolute fracture risk by age and bone mineral density}

In 2005, Osteoporosis Canada recommended identifying the absolute fracture risk by integrating the key risk factors for fracture (i.e., age, BMD, prior fracture, glucocorticoid use) in determining the Io-year risk of fragility fracture to be high (a risk of future fracture of $>20 \%)$, moderate $(\mathrm{IO} \%-20 \%)$ or low

Box 2: Common secondary causes of bone loss

- Hyperparathyroidism (primary or secondary)

- Vitamin D inadequacy

- Malabsorption state (e.g., celiac disease, inflammatory bowel disease, short gut syndrome)

- Hypercalciuria

- Hyperthyroidism

- Chronic lung disease

- Malignancy (e.g., myeloma, bony metastasis)

- Rheumatoid arthritis

- Hepatic insufficiency

Table 1: Interpretation of bone mineral density $(B M D)$ in men

\begin{tabular}{cl}
\hline Age group and score* $^{*}$ & \multicolumn{1}{c}{ Diagnostic classification } \\
\hline $\begin{array}{c}\text { Men } \geq 50 \text { yr: T-score } \\
\leq-2.5\end{array}$ & Osteoporosis \\
-1.5 to -2.5 & Reduced bone density \\
\hline Men $<50$ yr: $z$ score & \\
$<-2.0$ & Below the expected range for age \\
$\geq-2.0$ & Within the expected range for age \\
\hline
\end{tabular}

*Scores represent the number of standard deviations that BMD is above or below the mean normal reference range for men, as follows:

- T-score - young mean range

- $z$ score - age-matched mean range
(< $10 \%) .{ }^{5}$ Fig. I summarizes the interplay of BMD and age. It can be seen that only at T-scores below -3 do men aged 75 years and older reach a high-risk category, whereas a similar T-score at the age of 60 confers only a moderate risk. ${ }^{5}$ This indicates the important effect of age on the gradient of fracture risk. The additional presence of key risk factors (a preexisting fragility fracture or glucocorticoid use) moves the patient one risk category higher. These guidelines were based upon Swedish data; a more comprehensive calculation of Ioyear absolute fracture risk, based on BMD, age, sex and other risk factors from several large databases, is anticipated soon from WHO. These absolute fracture-risk data will help physicians to decide which patients might require prolonged treatment to reduce their risk of future fractures.

\section{How should osteoporosis be investigated in men?}

Evaluation includes a detailed history and physical examination to identify factors that may contribute to osteoporosis and to exclude secondary causes of bone loss (Box 3). Men should have their height measured at baseline and at serial assessments: height loss may reflect underlying vertebral compression fractures. Patients with back pain, height loss or kyphosis should undergo lateral spinal radiography to detect the presence of vertebral fractures.

Osteoblast and osteoclast activity can be assessed by measuring biochemical markers of bone turnover. Osteoblasts secrete noncollagenous proteins such as osteocalcin and bonespecific alkaline phosphatase, which can be measured in the serum. Carboxy- or amino-extension peptides of the procollagen molecule (P1CP, P1NP) also reflect bone formation rates, although these peptides can also be produced by other tissues (e.g., skin) and are not specific to bone. Bone resorption releases collagen degradation products such as amino- and carboxy-telopeptides of collagen cross-links (NTx, CTx) into the circulation, which are cleared renally. Markers of bone resorption can therefore be measured in serum or urine.

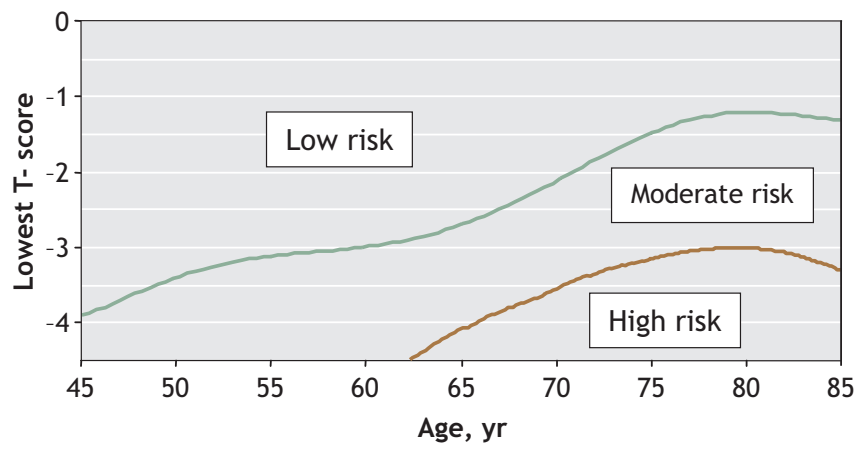

Fig. 1: Ten-year absolute risk of fracture for the low-, moderateand high-risk groups. The presence of a prior fragility fracture or glucocorticoid use increases the fracture risk into the next risk category. Reproduced, with permission, from the Canadian Association of Radiologists (Can Assoc RadiolJ 2005; 56:178-88). ${ }^{5}$ 
Although these biochemical markers have contributed to our knowledge of the pathophysiology and treatment of osteoporosis, they are subject to considerable biological variance in individuals, and at this time are not recommended in the clinical assessment of men with osteoporosis.

\section{Who should be treated?}

Pharmacological intervention is recommended for those at highest risk of a fragility fracture. Therapy should be considered for 4 groups of patients: ${ }^{4}$

- Men aged 65 years or older with a T-score less than -2.5 (at any measured site)

- Men aged 50 years or older with a fragility or vertebral compression fracture and a T-score less than -I.5

Box 3: Laboratory tests for the assessment of men with osteoporosis

Tests to exclude secondary causes of bone loss

- Complete blood count

- Serum calcium

- Albumin

- Liver transaminases

- Serum creatinine and calculated creatinine clearance

- Alkaline phosphatase

- Thyroid-stimulating hormone (TSH)

- Testosterone - total, as well as free or bioavailable

Additional tests, as suggested by results of clinical evaluation

- Parathyroid hormone (PTH)

- Serum 25-hydroxy vitamin D

- Serum immunoelectrophoresis

- Celiac antibody testing: gliadin, endomyseal, tissue transglutaminase

- 24-hour urine: calcium

- 24-hour urine: free cortisol
- Men of any age who are receiving glucocorticoid therapy for 3 months or more and a T-score less than -I.5

- Men of any age with clinical hypogonadism (from any cause) and a T-score less than -I.5

\section{How should osteoporosis in men be treated?}

Lifestyle and nutritional recommendations for the treatment of osteoporosis are similar for men and women. ${ }^{4}$ Table 2 outlines the various recommendations for managing osteoporosis in men.

Although clinical trial data on the efficacy of antiresorptive therapy in idiopathic osteoporosis in men are limited, the best evidence supports a primary role for bisphosphonates. ${ }^{6}$ Alendronate has Canadian regulatory approval for the treatment of osteoporosis in men, based on the results of a clinical trial ${ }^{6}$ involving 24I men with a fragility fracture or T-score at the femoral neck of -2 or less. The treatment groups received daily doses of alendronate (Io $\mathrm{mg}$ ), placebo, or calcium carbonate $(500 \mathrm{mg}$ ) and vitamin D (400 IU). After 24 months of therapy, the trial found bone density increases in the lumbar spine of an average of $7.1 \%$ in the treatment group versus $1.8 \%$ in the group taking calcium and vitamin $\mathrm{D}$ alone. Efficacies in eugonadal and hypogonadal men were equivalent. Recently, an open-label trial ${ }^{7}$ showed that daily 5 -mg doses of of risedronate were also effective in reducing vertebral fractures, by $60 \%$ in men with primary or secondary osteoporosis within I2 months of starting therapy. Both alendronate and risedronate are effective in the prevention and treatment of glucocorticoid-induced osteoporosis in both men and women, with improvements in BMD and reduction in fracture risk. ${ }^{8,9}$ Men with glucocorticoid-induced osteoporosis treated with risedronate have fewer vertebral fractures than those taking a placebo. ${ }^{9}$ Cyclical etidronate is also effective in preventing glucocorticoid-induced bone loss in men and women. ${ }^{10}$

Table 2: Treatment of osteoporosis, with grades of evidence, in men

\begin{tabular}{|c|c|c|c|}
\hline Drug & Dosage & Grade* & Contraindications and side effects \\
\hline \multicolumn{4}{|c|}{ Nutrients, total daily intake (diet + supplements) } \\
\hline Calcium & $1500 \mathrm{mg}$ & C & Contraindications: hypercalcemia, hypercalciuria \\
\hline Vitamin $\mathrm{D}_{3}$ (cholecalciferol) & $\geq 800 \mathrm{IU}$ & A & \\
\hline $\begin{array}{l}\text { Bisphosphonate therapy: } \\
\text { alendronate } \dagger \\
\text { or risedronate } \dagger \\
\text { or cyclical etidronate } \neq\end{array}$ & $\begin{array}{c}70 \mathrm{mg} / \mathrm{wk} \\
35 \mathrm{mg} / \mathrm{wk} \\
400 \mathrm{mg} / \mathrm{d} \text { for } 14 \mathrm{~d} \text { per } 90-\mathrm{d} \text { cycle }\end{array}$ & A & $\begin{array}{l}\text { Contraindications: renal failure (glomerular filtration rate } \\
<30 \mathrm{~mL} / \mathrm{min} \text { ), history of allergy to bisphosphonate exposure } \\
\text { Side effects: usually limited to } \mathrm{Gl} \text { intolerance }\end{array}$ \\
\hline Anabolic therapy: teriparatide & $\begin{array}{l}20 \mu \mathrm{g} / \mathrm{d} \text { subcutaneously } \\
\text { for } 18 \mathrm{mo}\end{array}$ & D§ & $\begin{array}{l}\text { Contraindications: skeletal malignancy, a history of radio- } \\
\text { therapy } 1 \text { of the skeleton, Paget's disease, hypercalcemia } \\
\text { Side effects: nausea, headaches, muscle cramps }\end{array}$ \\
\hline
\end{tabular}

*Grades of evidence, from the 2002 Osteoporosis Canada Guidelines.

†For treatment of idiopathic osteoporosis, or for treatment and prevention of glucocorticoid osteoporosis.

fFor prevention (not treatment) of glucocorticoid osteoporosis.

§Although the randomized controlled trials (RCTs) that involved men were not powered to detect reductions in fracture incidence, the pivotal RCT (evidence grade A),

which involved postmenopausal women with severe osteoporosis, resulted in significant reductions in both vertebral and nonvertebral fractures.

IInternal or external. 
Testosterone therapy over a period of 3-4 years in hypogonadal men improves spine and hip BMD, although no trials have demonstrated that fracture risk is reduced. Testosterone may be most appropriate for men with symptoms of hypogonadism (e.g., sexual dysfunction, anemia), in whom testosterone may provide additional, extraskeletal benefits.

Subcutaneous teriparatide (recombinant human parathyroid hormone I-34) has recently been approved in Canada for the management of osteoporosis: significant improvements in BMD have been observed in both men as well as women. ${ }^{11}$ Vertebral and nonvertebral fracture benefit was documented only in postmenopausal women, however, because trials that involved men were not powered for fracture outcomes. Osteoporosis Canada has prepared clinical guidelines for teriparatide treatment for patients with osteoporosis. ${ }^{12}$

No randomized clinical trials of calcitonin use in men have been reported.

\section{Case revisited}

Because this patient had height loss and multiple risk factors for fracture (age, glucocorticoid use), spinal radiographs were performed. Fractures were found at T8 and Tio. His BMD was assessed with dual-energy $\mathrm{x}$-ray absorptiometry (DXA) for additional information on fracture risk. Given his $\mathrm{T}$-score of -2.5 , previous vertebral fractures and long-term use of glucocorticoids, his ro-year absolute risk of fracture is high ( $\geq 20 \%$ probability; Fig. I).

After other secondary causes of bone loss were excluded, a diagnosis was made of glucocorticoid-induced osteoporosis. Appropriate management included lifestyle changes, calcium and vitamin D supplementation, and bisphosphonate therapy. The DXA scan also serves as a baseline for serial progress: a repeat BMD assessment was scheduled after I year to ensure that his bone mass was stabilized.

Osteoporosis is underdiagnosed in older men, despite its significant association with disability and death. With appropriate management, the fracture burden can now be reduced.

\section{This article has been peer reviewed.}

From the Divisions of Endocrinology and Geriatrics (Khan) and Geriatric Medicine (Papaioannou), Department of Medicine, McMaster University, Hamilton; Divisions of Endocrinology and Nephrology (Hodsman), Department of Medicine, University of Western Ontario, London, Ont.; Division of Endocrinology (Kendler), Department of Medicine, University of British Columbia, Vancouver, BC; Division of Rheumatology (Brown), Department of Clinical Medicine, Laval University, Quebec City, Que.; and Division of Rheumatology (Olszynski), Department of Clinical Medicine, University of Saskatchewan, Saskatoon, Man.
Competing interests: None declared by Wojciech Olszynski; all coauthors stated that their opinions have been uninfluenced by external agencies or sources. Aliya Khan has received consultancy fees, research grants and/or honoraria from Aventis-Sanofi, Merck, Eli Lilly, Novartis, NPS Allelix, Servier, and Proctor \& Gamble. Anthony Hodsman has received consultancy fees, research grants and honoraria from Eli Lilly, Merck Frosst, NPS Allelix, Zelos Therapeutics, Servier and Proctor \& Gamble/Sanofi Aventis. Alexandra Papaioannou has received grants, honoraria and travel assistance from Eli Lilly, Amgen, Merck, Novartis, Procter \& Gamble and Aventis-Sanofi, including for participation in the development of this article. David Kendler has received research grants, consultancy fees and lecture honoraria from Merck, Eli Lilly, Novartis, Wyeth, Pfizer, Servier, Takeda, Amgen, NPS Allelix and Zelos. Jacques Brown has received consultancy and speaker's fees from Procter \& Gamble, Sanofi-Aventis, Eli Lilly and Novartis.

Contributors: Aliya Khan was responsible for coordinating the writing and content of the manuscript. All coauthors contributed to the writing of the paper and coordinated input from Osteoporosis Canada.

Acknowledgements: This work was supported by unrestricted grants to Osteoporosis Canada by Merck Frosst Canada, Organon, Canada, and Procter \& Gamble Pharmaceuticals, Canada, which provided partial funding to cover ancillary costs of preparing this paper (e.g., face-to-face meetings). The donors had no influence on the content of the paper, nor any opportunity to review it before it was submitted to $C M A J$.

\section{REFERENCES}

I. Khan AA, Bachrach L, Brown JP, et al. Standards and guidelines for performing central dual-energy x-ray absorptiometry in premenopausal women, men, and children. JClin Densitom 2004;7:5I-64.

2. Jackson SA, Tenenhouse A, Robertson L. Vertebral fracture definition from population-based data: preliminary results from the Canadian Multicenter Osteoporosis Study (CaMos). Osteoporos Int 2000;1I:680-7.

3. Johnell O, Kanis J, Gullber G. Mortality, morbidity and assessment of fracture risk in male osteoporosis. Calcif Tissue Int 2001;69:182-4.

4. Brown JP, Josse RG; Scientific Advisory Council of the Osteoporosis Society of Canada. 2002 clinical practice guidelines for the diagnosis and management of osteoporosis in Canada [review. Published errata in $C M A J$ 2003;18;168(4):400, 2003;18;I68(5):544 and 2003;I8;I68(6):676]. CMAJ 2002;I67 (Io Suppl):SI-34.

5. Siminoski K, Leslie WD, Frame H, et al. Recommendations for bone mineral density reporting in Canada. Can Assoc Radiol J 2005;56:178-88.

6. Olszynski WP, Shawn DK, Adachi JD, et al. Osteoporosis in men: epidemiology, diagnosis, prevention, and treatment. Clin Ther 2004;26:15-28.

7. Ringe JD, Faber H , Farahmand P, et al. Efficacy of risedronate in men with primary and secondary osteoporosis: results of a I-year study. Rheumatol Int 2006;26: 427-3I.

8. Saag KG, Emkey R, Schnitzer TJ, et al. Alendronate for the prevention and treatment of glucocorticoid-induced osteoporosis. N Engl J Med I998;339:292-9.

9. Reid D, Hughes R, Laan RF, et al. Efficacy and safety of daily risedronate in the treatment of corticosteroid-induced osteoporosis in men and women: a randomized trial. J Bone Miner Res 2000; I5:1006-I3.

Io. Adachi JD, Bensen WG, Brown J, et al. Intermittent etidronate therapy to prevent corticosteroid-induced osteoporosis. NEngl J Med I997;337:382-7.

II. Cranney A, Papaioannou A, Zytaruk N, et al. Parathyroid hormone for the treatment of osteoporosis: a systematic review. CMAJ 2006;175:52-9.

I2. Hodsman A, Papaioannou A, Cranney A. Clinical practice guidelines for the use of parathyroid hormone in the treatment of osteoporosis. CMAJ 2006;175:52-9.

Correspondence to: Dr. Aliya Khan, Professor of Clinical Medicine, Divisions of Endocrinology and Geriatrics, McMaster University, 33I Sheddon Ave., Ste. 209, Oakville ON L6J IX8; fax 905 844-8966; aliya@mcmaster.ca 